\title{
Nuclear localization of beta-catenin involved in precancerous change in oral leukoplakia
} Kosei Ishida ${ }^{1}$, Satoshi Ito ${ }^{1}$, Naoyuki Wada ${ }^{2}$, Hiroyo Deguchi ${ }^{1}$, Tsuyoshi Hata ${ }^{1}$, Masaru Hosoda ${ }^{1}$ and Tsutomu Nohno*2

\author{
Address: ${ }^{1}$ Department of Oral Surgery, Kawasaki Medical School, Kurashiki 701-0192, Japan and ${ }^{2}$ Department of Molecular and Developmental \\ Biology, Kawasaki Medical School, Kurashiki 701-0192, Japan \\ Email: Kosei Ishida - kishida@med.kawasaki-m.ac.jp; Satoshi Ito - hibitan@med.kawasaki-m.ac.jp; Naoyuki Wada - wada@med.kawasaki- \\ m.ac.jp; Hiroyo Deguchi - deguchi@med.kawasaki-m.ac.jp; Tsuyoshi Hata - denthata@med.kawasaki-m.ac.jp; \\ Masaru Hosoda - chou@med.kawasaki-m.ac.jp; Tsutomu Nohno* - nohno@bcc.kawasaki-m.ac.jp \\ * Corresponding author
}

Published: 9 October 2007

Molecular Cancer 2007, 6:62 doi:10.1 I86/1476-4598-6-62
Received: 23 August 2007

Accepted: 9 October 2007

This article is available from: http://www.molecular-cancer.com/content/6/1/62

(c) 2007 Ishida et al; licensee BioMed Central Ltd.

This is an Open Access article distributed under the terms of the Creative Commons Attribution License (http://creativecommons.org/licenses/by/2.0), which permits unrestricted use, distribution, and reproduction in any medium, provided the original work is properly cited.

\begin{abstract}
Background: Oral leukoplakia is a precancerous change developed in the oral mucosa, and the mechanism that oral leukoplakia becomes malignant through atypical epithelium is not known. Here we compared the $\beta$-catenin expression detected by immunohistochemical staining in the normal oral epithelium and in the oral leukoplakia with or without dysplasia.
\end{abstract}

Results: The normal oral epithelium showed $\beta$-catenin expression only in the cell membrane, but not in the nuclei. In the oral leukoplakia without dysplasia, 7 out of 17 samples $(41 \%)$ showed $\beta$ catenin expression in the cell membrane, and 5 samples $(29 \%)$ showed expression in the nuclei. In the oral leukoplakia with dysplasia, nuclear expression of $\beta$-catenin was shown in 11 out of 12 samples (92\%). Incidence of nuclear $\beta$-catenin expression was significantly different between dysplasia and normal oral epithelium $(P<0.0 \mathrm{I})$, and also between oral leukoplakia with dysplasia and those without dysplasia $(P<0.01)$. Wnt3 expression was detected in the epithelial cell membrane or cytoplasm in oral leukoplakia where nuclear expression of $\beta$-catenin was evident, but not in epithelial cells without nuclear expression of $\beta$-catenin.

Conclusion: The components of canonical Wnt pathway, such as Wnt3, $\beta$-catenin, and cyclin DI, were detected, implying that this pathway is potentially involved in the progression of dysplasia in oral leukoplakia.

\section{Background}

Oral leukoplakia is known as a precancerous change of squamous cell carcinoma developed in oral mucosa [1-3]. Leukoplakia is a term expressing clinical disease state, and it occurs in every intra-oral locus and shows various observations. Because a clinician is difficult to be settled with precancerous lesion in these, we require histopathology examination. Leukoplakia diagnosed as epithelial dyspla- sia histopathology in leukoplakia becomes precancerous. It is said that it may become malignant transformation in progression of the severity in epithelial dysplasia [4]. Although much works have been done on the leukoplakia, the mechanism that oral mucosa epithelium constituting leukoplakia becomes malignant through atypical epithelium is not known. 
There are many reports on $\beta$-catenin accumulation into a nucleus of a cancer cell in the epithelial malignant tumor, including colorectal cancer [5-8], and transcription activation of a target gene by $\beta$-catenin/TCF is known to participate in malignant transformation $[9,10]$. Transcription activation by $\beta$-catenin is triggered by binding of Wnt family to the cell membrane receptors, called Frizzled and LRP5/6, which transduces Wnt signal inside the cell through inactivation of GSK-3. APC and Axin constitute a complex to phosphorylate $\beta$-catenin with GSK-3. In the presence of Wnt, the receptor complex transduces negative signals to APC/Axin/GSK-3 complex through Dishevelled, and thus GSK-3 becomes unable to phosphorylate cellular $\beta$-catenin. Accumulated $\beta$-catenin in cytoplasm is now translocated into a nucleus, forms a transcriptional activation complex with TCF/LEF1 [10-13], and activates various target genes such as c-myc and cyclin D1 [14-17]. In the absence of Wnt, $\beta$-catenin in cytoplasm is immediately phosphorylated by GSK-3/Axin/APC complex and receives ubiquitination, eventually leading to degradation in proteasome, and thus disappeared in cytoplasm immediately $[9,10]$.

Because Wnt family is known to participate in epithelial cell proliferation, we examined in this study immunohistochemical localization of $\beta$-catenin, with attention to transcription activation of a target gene by $\beta$-catenin, and to evaluate nuclear accumulation $\beta$-catenin in relevance with atypical epithelium in a surface layer of oral leukoplakia. Nuclear localization of $\beta$-catenin is correlated with cyclin D1 expression in oral leukoplakia, and also Wnt3 expression in neighboring cells, known as a typical member of the Wnt family that activates $\beta$-catenin-mediated signaling $[18,19]$, suggesting the involvement of $\mathrm{Wnt} / \beta$ catenin signaling in the progression of dysplasia.

\section{Results}

\section{Immunohistochemical localization of $\beta$-catenin}

The expression patterns of $\beta$-catenin in oral mucosa are summarized in Table 1 . In the normal oral epithelium, nuclear localization of $\beta$-catenin was not detected in all 6 samples examined; 5 out of 6 samples showed expression signals only in the cell membrane, and one sample showed cytoplasmic expression in addition to signals in the cell membrane. In the oral leukoplakia without dysplasia, 7 out of 17 samples (41\%) showed expression signals in the cell membrane, and 5 samples (29\%) showed expression signals in the nuclei. Oral leukoplakia with dysplasia did not show expression signals in the cell membrane, and nuclear expression was shown in 11 out of 12 samples $(92 \%)$.

Localization of $\beta$-catenin in the epithelial cell membranes was observed in normal oral epithelium and oral leukoplakia, whereas expression in OSCC was low or totally absent in the cell membrane (Fig. 1). The expression of $\beta$ catenin in normal oral epithelium was observed on the cell membrane, but not within the nuclei of basal and spinous layer (Fig. 1b, 1c). In oral leukoplakia without dysplasia, the expression of $\beta$-catenin was observed on the cell membrane or both cell membrane and cytoplasm in $24 \%$ of basal and spinous layer cells (Fig. 1e, 1f). In oral leukoplakia with mild dysplasia, the expression of $\beta$-catenin was observed in the nuclei at about $30 \%$ (Fig. 1h, 1i). Oral leukoplakia with dysplasia that was especially characterized by an increased nuclear-cytoplasmic ratio, an increased number of mitotic figures, including abnormal mitoses, nuclear hyperchromatism showed nuclear expression pattern (Fig. 1k, 1l), and the expression of $\beta$ catenin in nuclei was shown in more than $80 \%$ of epithelial cells. The mean percentage of nuclear staining with $\beta$ catenin in oral leukoplakia is presented in Table 2. The nuclear expression of $\beta$-catenin in epithelial dysplasia increased depending on the grade of dysplasia, and there were significantly different between $\beta$-catenin staining without dysplasia and that with mild dysplasia $(P<0.01)$, and also between $\beta$-catenin staining with mild dysplasia and that with severe dysplasia $(\mathrm{P}<0.05)$. The tumor parenchymal cells of OSCC (Fig. $1 \mathrm{~m}-\mathrm{O}$ ) also showed nuclear expression pattern in 10 out of 15 specimens $(67 \%)$. Incidence of nuclear localization of $\beta$-catenin was significantly different between dysplasia and normal oral epithelium $(\mathrm{P}<0.01)$, and also between oral leukoplakia with dysplasia and those without dysplasia $(\mathrm{P}<0.01)$.

Table I: Expression patterns of $\beta$-catenin in oral mucosa

\begin{tabular}{|c|c|c|c|c|}
\hline Tissue & Total number & Cytoplasm & Nucleus & Membrane \\
\hline Normal oral epithelium & 6 & 1 & 0 & 5 \\
\hline \multicolumn{5}{|l|}{ Oral leukoplakia } \\
\hline Without dysplasia & 17 & 5 & 5 & 7 \\
\hline With dysplasia & 12 & 1 & $11 *$ & 0 \\
\hline Oral squamous cell carcinoma & 15 & 5 & 10 & 0 \\
\hline
\end{tabular}

$* \mathrm{P}<0.0$ I between normal oral epithelium and oral leukoplakia without dysplasia by Fisher's exact test. 

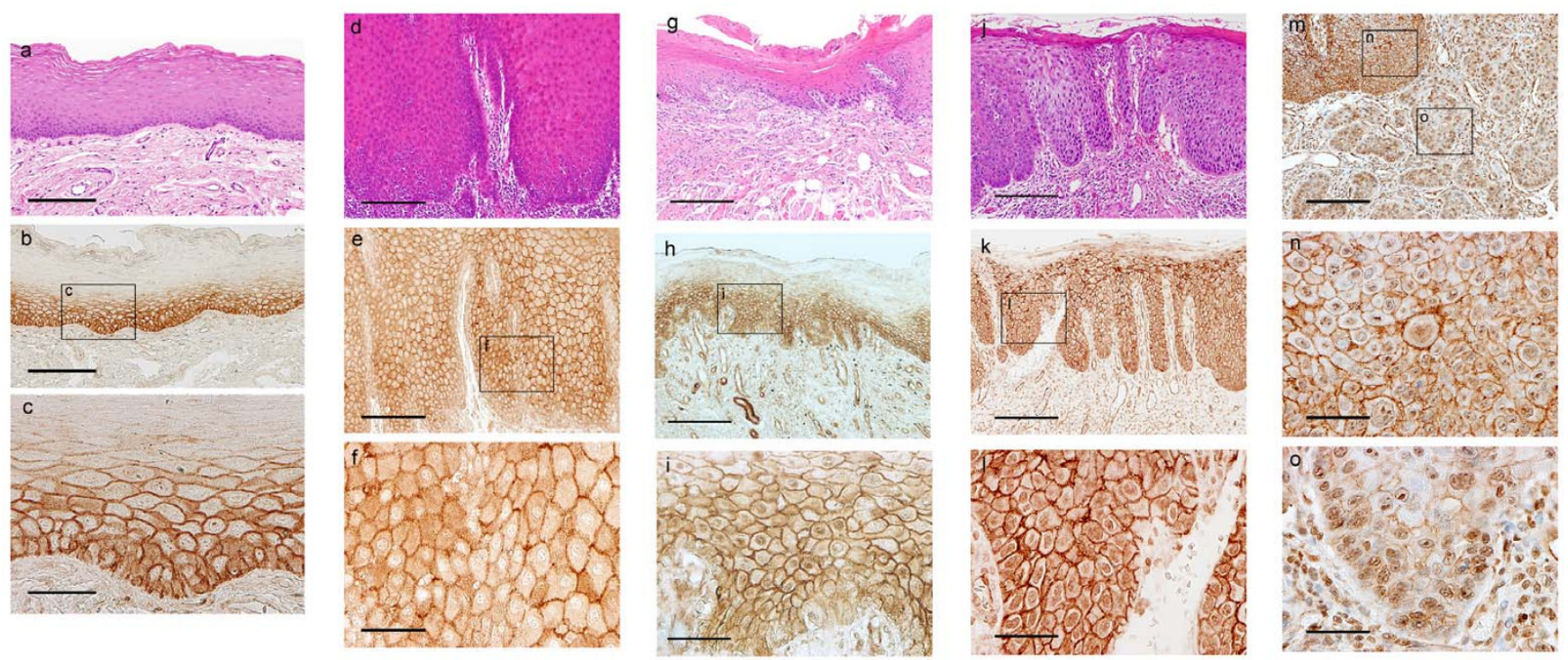

\section{Figure I}

Immunohistochemical localization of $\beta$-catenin in normal oral epithelium (a-c), oral leukoplakia without dysplasia (d-f), oral leukoplakia with mild dysplasia $(g-i)$, oral leukoplakia with severe dysplasia $(j-l)$, and oral squamous cell carcinoma $(m-o)$. $(a, d, g, j)$ Hematoxylin and eosin staining. (b, c, e, f, h, i, k, l, m-o) $\beta$-Catenin staining. (b, c) Signals were detected in the cell membrane of the basal and spinous layer, but not in the cytoplasm and nuclei. (e, f) Signals were detected in the cell membranes and cytoplasm. ( $h$, i) Signals were detected in the cell membranes and nuclei. (j) The area for dysplasia is characterized by an increased nuclear-cytoplasmic ratio, an increased number of mitotic figures, including abnormal mitoses, nuclear hyperchromatism. (k, l) Signals were detected in the cell membranes and nuclei. $(\mathrm{m}-\mathrm{o})$ Signals were detected in the nucleus of the epithelial dysplastic cells $(n)$ and carcinoma cells (o) in OSCC, but cell membranous expression was weak or absent. Scale bars: (a, b, d, e, g, h, j, k, m) $200 \mu \mathrm{m} ;(\mathrm{c}, \mathrm{f}, \mathrm{i}, \mathrm{l}, \mathrm{n}, \mathrm{o}) 50 \mu \mathrm{m}$.

\section{Relationship between localization of $\beta$-catenin and Wnt3 expression}

We examined the expression pattern of Wnt3 in oral leukoplakia. Wnt3 was not expressed in the normal oral epithelium (data not shown). Figure 2 shows comparison of immunohistochemical staining for $\beta$-catenin, Wnt3, cyclin D1, and c-myc in oral leukoplakia without dysplasia (a-d) and those with dysplasia (e-l). Wnt3 was expressed on the epithelial cell membrane or cytoplasm where nuclear expression of $\beta$-catenin is evident, in contrast to those without nuclear expression of $\beta$-catenin (Fig. 2b, 2f, $2 \mathrm{j})$. In the samples with nuclear expression of $\beta$-catenin,

Table 2: Mean percentage of nuclear $\beta$-catenin staining in oral leukoplakia with and without dysplasia

\begin{tabular}{lcc}
\hline Pathological diagnosis & Total number & Positive ratio (mean value) \\
\hline No dysplasia & 17 & 6.7 \\
Mild dysplasia & 9 & $26.9 *$ \\
Severe dysplasia & 3 & $59.7^{* *}$
\end{tabular}

*P $<0.0$ I between no dysplasia samples and mild dysplasia samples by Student's $t$-test.

**P $<0.05$ between mild dysplasia samples and severe dysplasia samples by Student's $t$-test.
Wnt3 expression was shown in 13 out of 16 samples $(81 \%)$, and there is significant positive correlation between the nuclear expression of $\beta$-catenin and Wnt3 staining $(\mathrm{P}<0.01)$ (Table 3$)$.

We also examined other members of Wnt family, including Wnt1, Wnt5a, and Wnt7a, for immunohistochemical staining. No signal was detectable with antibodies against Wnt 1 and Wnt7a in normal and leukoplakia epithelia, whereas Wnt5a signal was ubiquitously detectable in normal oral epithelium, leukoplakia, and in OSCC (data not shown). Thus, these Wnt members are unlikely to be involved in nuclear localization of $\beta$-catenin because of the absence of correlation.

\section{Relationship with cyclin DI and c-myc expression}

Cyclin D1 was intensely expressed in the oral leukoplakia where nuclear expression of $\beta$-catenin is evident, in contrast to those without nuclear expression of $\beta$-catenin (Fig. 2). Cyclin D1 overexpression was more evident in the oral leukoplakia with dysplasia than that without dysplasia (Fig. 2c, 2g, 2k), and percentage of the cell number with positive nuclear staining is significantly different in these specimens (Fig. 3). On the other hand, the c-myc expres- 

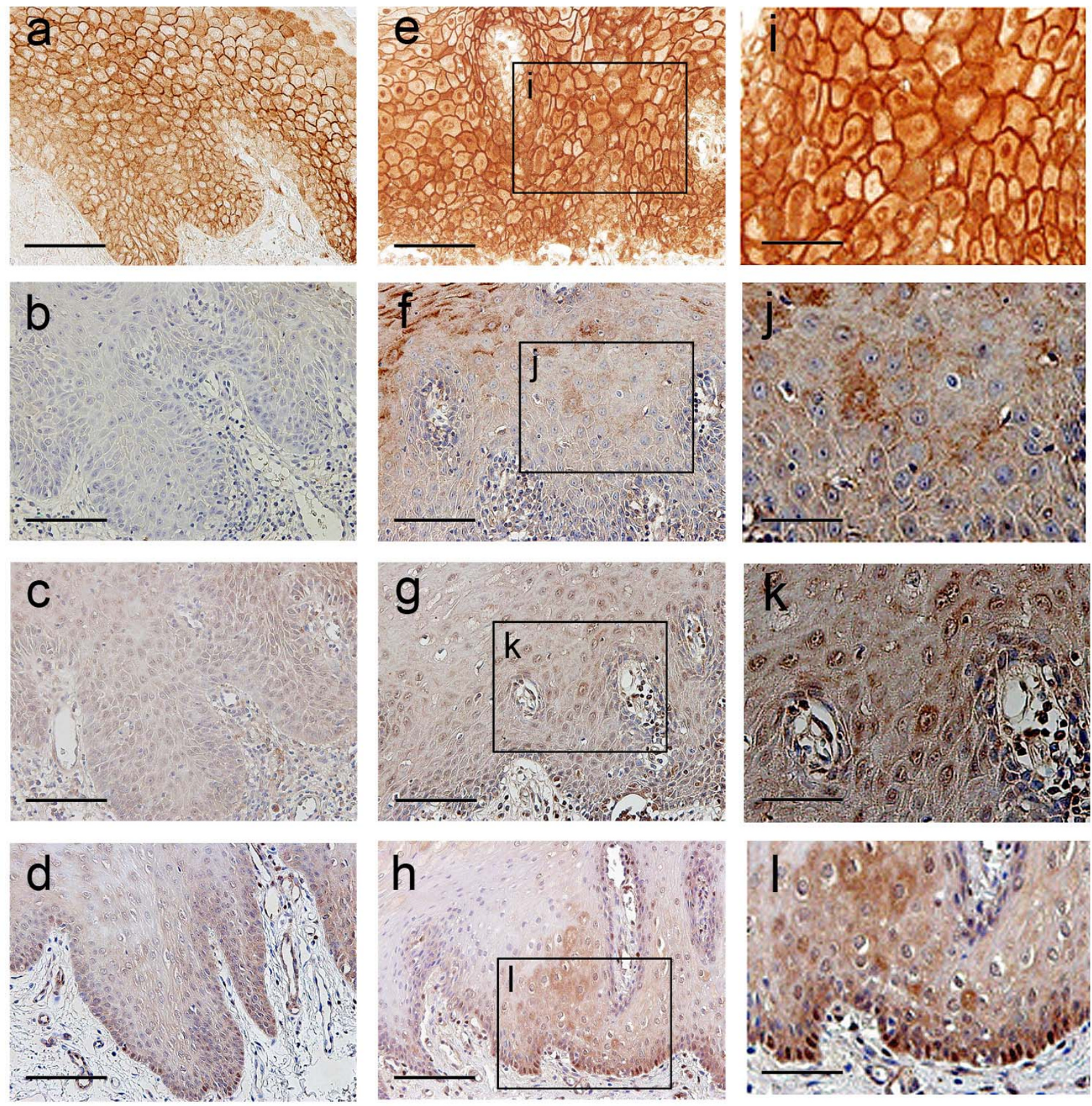

\section{Figure 2}

Comparison of immunohistochemical staining for $\beta$-catenin, Wnt3, cyclin DI, and c-myc in oral leukoplakia. (a-d) Serial sections of oral leukoplakia without dysplasia. (a) Nuclear expression of $\beta$-catenin was not observed. (b) Wnt3 expression was not observed. (c) Cyclin DI shows weak expression. (d) Nuclear expression of c-myc is observed in the basal layer cells. (e-h) Serial sections of oral leukoplakia with dysplasia. (e, i) $\beta$-Catenin is expressed in the nuclei. (f, j) Wnt3 expression is observed on the epithelial cell membrane and in the cytoplasm. $(\mathrm{g}, \mathrm{k})$ Cyclin DI is expressed in several epithelial cells. (h, I) c-Myc shows similar expression pattern as oral leukoplakia without dysplasia. Scale bars: (a-h) $100 \mu \mathrm{m}$; (i-l) $50 \mu \mathrm{m}$. 
Table 3: Relationship between nuclear expression of $\beta$-catenin and $W n t 3$ expression in oral leukoplakia

\begin{tabular}{lccc}
\hline$\beta$-Catenin & Total number & & Wnt3 \\
\cline { 3 - 4 } & & - & + \\
\hline Nuclear expression negative & 13 & 10 & $3(23.1 \%)$ \\
Nuclear expression positive & 16 & 3 & $13(81.3 \%)^{*}$ \\
\hline
\end{tabular}

$* \mathrm{P}<0.0 \mathrm{I}$ between nuclear expression negative and positive samples by Mann-Whitney U-test.

sion in nuclei was not significantly different between these two specimens (Fig. 2d, 2h, 2l), suggesting independent activation of c-myc.

\section{Discussion}

$\beta$-Catenin has been identified to be a constituent of cell adhesion apparatus bound to cadherin family [20], and plays an important role for cellular movement and adhesion, as well as a signaling factor involved in canonical Wnt pathway [9]. Nuclear localization of $\beta$-catenin is known to associate with malignant transformation of colorectal cancer and other tumor [5-8]. Because cytoplasmic accumulation and subsequent nuclear translocation of $\beta$-catenin is known to be resulted from activation of canonical Wnt signaling pathways [10], subcellular localization of $\beta$-catenin is a useful marker to detect cellular conditions to proliferate actively. We compared here immunohistochemical localization of $\beta$-catenin in the

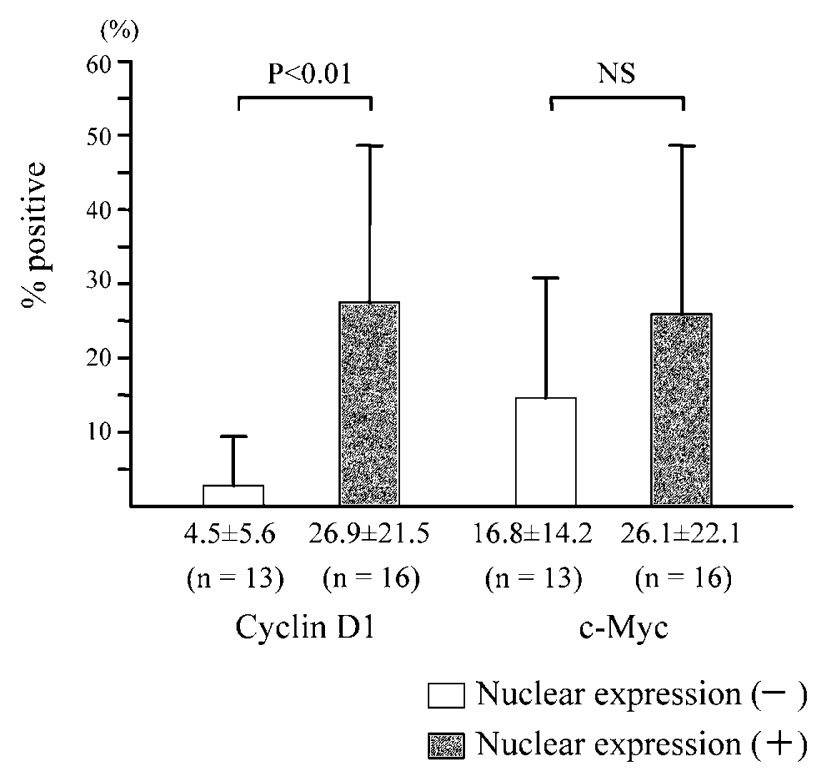

Figure 3

Relationship between subcellular localization of $\beta$-catenin and expression patterns of cyclin DI and c-myc in oral leukoplakia. Shown by means with standard deviations. normal oral epithelia, oral leukoplakia, and OSCC, aimed at elucidating relationship between proliferative activity and subcellular localization of the products.

In contrast to membranous and cytoplasmic expression, nuclear expression of $\beta$-catenin is implicated in tumor progression, but the significance of $\beta$-catenin localization in the oral epithelial dysplasia and OSCC has not yet been examined in details. The nuclear expression of $\beta$-catenin increased during progression of the severity in oral leukoplakia. Furthermore, because localization of $\beta$-catenin in normal oral epithelium and dysplasia showed significant difference, suggesting nuclear expression of $\beta$-catenin as aberrant condition. In addition, nuclear expression of $\beta$ catenin was also observed in 10 out of 15 samples of OSCC, and membranous $\beta$-catenin expression was low or totally absent in 7 out of 15 samples of OSCC (data not shown). In various malignant tumors, reduced membranous $\beta$-catenin expression was associated with the emergence of invasion or metastasis [21-24]. Our results suggest that $\beta$-catenin function in cell-cell adhesion and translocation in the nucleus are related to proliferation and invasion of OSCC.

c-Myc and cyclin D1 have been identified as target genes of the Wnt/ $\beta$-catenin pathway [14-17]. Tetsu and McCormick [25] reported that expression of cyclin D1 is strongly dependent on $\beta$-catenin/TCF and has a direct effect on cell proliferation in colon carcinoma cells. Furthermore, Kovesi and Szende [26] reported that expression of cyclin D1 increased during progression of the severity in oral leukoplakia. Our results showed elevated cyclin D1 expression in the epithelial dysplasia. In oral epithelium, cyclin D1 may participate in malignant transformation. cMyc expression was elevated in dysplasia with nuclear expression of $\beta$-catenin than that without nuclear expression, although the difference in c-myc expression is not evident.

Our studies show the aberrant Wnt signaling pathway in the oral epithelial dysplasia. Wnt3, a typical member of the Wnt family that activates $\beta$-catenin mediated signaling $[18,19]$, was not expressed in the normal oral epithelium. In contrast, Wnt3 expression was observed in the examples showing nuclear expression of $\beta$-catenin. Both the nuclear expression of $\beta$-catenin and Wnt 3 expression were observed in oral leukoplakia with dysplasia, and therefore the aberrant Wnt signaling pathway may promote malignant transformation by triggering cyclin D1 expression and consequently uncontrolled progression into the cell cycle.

These lines of evidence suggest that nuclear accumulation of $\beta$-catenin plays an important role during malignant transition of oral leukoplakia through dysplasia. In addi- 
tion to pathological examination of hematoxylin and eosin-stained sections for oral leukoplakia, immunochemical staining for $\beta$-catenin is useful for diagnosis of epithelial dysplasia and precancerous changes. Evaluation of other constituents for Wnt signaling, including GSK-3, CDK, Frizzled, and LRP5/6, helps us for determination and prognosis of precancerous change of dysplasia.

\section{Conclusion}

As for the transcriptional activation of a target gene by $\beta$ catenin, elevated expression was detected in a squamous cell carcinoma and epithelial dysplasia of oral leukoplakia. Thus, Wnt/ $\beta$-catenin pathway is considered to be involved in the progression of dysplasia in oral leukoplakia, as shown by nuclear expression of $\beta$-catenin and other components, including Wnt3 and cyclin D1.

\section{Methods}

\section{Tissue samples}

Formalin-fixed, paraffin-embedded samples of normal oral mucosa (6 examples), of oral squamous cell carcinoma (15 examples), and of oral leukoplakia (29 examples) were used. A fresh tissue of the oral cavity is also obtained at the time of biopsy or at oral surgery during treatment of cancerous disease after obtaining informed consent to the patients, and fixed in formalin before paraffin embedding. A histopathological diagnosis of each sample was performed by pathological staffs in the Department of Pathology in Kawasaki Medical School Hospital. According to the WHO diagnostic criteria for histological typing of cancer and pre-cancer of the oral mucosa [1], 12 examples from oral leukoplakia were diagnosed as epithelial dysplasia lesion (dysplasia). Dysplasia was diagnosed and graded as mild dysplasia ( 9 examples) and severe dysplasia (3 examples).

\section{Immunohistochemistry}

Sections of $4 \mu \mathrm{m}$ thickness in paraffin embedding tissue were treated with microwave for 10 minutes in $10 \mathrm{mM}$ citrate buffer ( $\mathrm{pH} \mathrm{6)}$ after de-paraffin processing, followed by washing with PBS twice for 10 minutes. To remove endogenous peroxidase activity, sections were treated in 3\% hydrogen peroxide solution for 15 minutes, and subsequently in PBS twice for five minutes. The primary antibodies against $\beta$-catenin (Sigma-Aldrich), Wnt3 (Santa Cruz Biotechnology), cyclin D1 (Zymed Laboratories), and c-myc (Santa Cruz Biotechnology) were used at $1 /$ $400,1 / 100,1 / 50$, and $1 / 50$ dilution, respectively, in PBS by incubating at room temperature for $60 \mathrm{~min}$. After washing with PBS, the sections were treated with biotinylated secondary antibody for $10 \mathrm{~min}$ and then with peroxidase-labeled streptavidin for 10 min (LSAB PLUS kit, DAKO, Japan), and the color was developed with 3,3'diaminobenzidine tetrahydrochloride (LSAB PLUS kit, DAKO, Japan), followed by counter staining with Mayer's hematoxylin. As a negative control, primary antibodies were omitted and the sections were incubated with the secondary antibody alone.

\section{Evaluation of staining}

For counting cells with nuclear and/or cytoplasm staining, three microscopic fields with 200-fold magnification were randomly chosen and the mean above $10 \%$ of the total cell numbers was deduced to be positive.

Evaluation of staining was carried out based on whether nuclear or cytoplasmic staining is detectable. Under microscopic field of 200-fold magnification, tissues are positive for $\beta$-catenin, if more than $10 \%$ of cells show cytoplasmic or nuclear staining. Randomly selected field in the tumor parenchymal region was used for determination of $\beta$-catenin positive cells in squamous cell carcinoma. Because signal intensity and distribution were different depending on the places in leukoplakia without dysplasia and in the normal oral epithelium, three fields were randomly selected to determine average ratio of a positive cell. In the Wnt 3 staining, the cell membranous or cytoplasmic staining in the basal and spinous layer was regarded as positive. For cyclin D1 and c-myc, nuclear staining was regarded as positive. The cyclin D1 and c-myc staining was evaluated by counting at 200 -fold magnification and calculating the percentage of positive cells. Statistical significance was estimated using Fisher's exact test, Mann-Whitney $U$-test, and Student's $t$-test.

\section{Abbreviations}

GSK-3 - glycogen synthase kinase-3; LEF1 - lymphoid enhancer binding factor 1; LRP - low-density lipoprotein receptor-related protein; OSCC - oral squamous cell carcinoma; PBS - phosphate-buffered saline; TCF - T cell factor.

\section{Competing interests}

The author(s) declare that they have no competing interests.

\section{Authors' contributions}

$\mathrm{KI}$ and SI carried out immunohistochemical analysis and evaluation of staining, KI also drafted the manuscript, NW participated in evaluation of immunohistochemical staining and helped to draft the manuscript, HD and TH participated to collect specimens and performed in the statistical analysis, MH and TN participated in the design and coordination of the study, TN also conceived of the study and helped to draft the manuscript. All authors read and approved the final manuscript.

\section{Acknowledgements}

The authors thank Dr. Takayoshi Honma and Dr. Naoya Kitamura, formerly in Kawasaki Medical School for helpful discussion, Dr. Isao Irei for his help in histological diagnosis, and Kazumi Wakabayashi and Yoko Yosh- 
ida for excellent technical assistance. This work is supported in part by Research Project Grants from Kawasaki Medical School (17-I0IN, I7$108 \mathrm{~N}, 18-102,19-102 \mathrm{~N}$, and 19-108N).

\section{References}

I. Pindborg JJ, Reichart PA, Smith CJ, van der Waal I: World Health Organization: histological typing of cancer and precancer of the oral mucosa. Berlin: Springer; 1997:25-26.

2. Silverman S Jr, Gorsky M, Lozada F: Oral leukoplakia and malignant transformation: A follow-up study of 257 patients. Cancer 1984, 53:563-568.

3. WHO Collaborating Center for Oral Precancerous Lesions: Definition of leukoplakia and related lesions: An aid to studies on oral precancer. Oral Surg Oral Med Oral Pathol 1978, 46:5।8-539.

4. Reibel J: Prognosis of oral pre-malignant lesion: significance of clinical, histopathological, and molecular biological characteristics. Crit Rev Oral Biol Med 2003, 14:47-62.

5. Morin PJ, Sparks AB, Korinek V, Barker N, Clevers H, Vogelstein B, Kinzler KW: Activation of beta-catenin-Tcf signaling in colon cancer by mutations in beta-catenin or APC. Science 1997, 275: $1787-1790$.

6. Ogasawara N, Tsukamoto T, Mizoshita T, Inada K, Cao X, Takenaka $Y$, Joh T, Tatematsu M: Mutations and nuclear accumulation of beta-catenin correlate with intestinal phenotypic expression in human gastric cancer. Histopathology 2006, 49:6I2-62I.

7. Takayama T, Shiozaki H, Shibamoto S, Oka H, Kimura Y, Tamura S, Inoue M, Monden T, Ito F, Monden M: Beta-catenin expression in human cancers. Am J Pathol I996, I 48:39-46.

8. Zhou XB, Lu N, Zhang W, Quan LP, Lin DM, Wang QH, Li YL, Xu NZ: Expression and significance of beta-catenin in esophageal carcinoma. Ai Zheng 2002, $21: 877-880$.

9. Li H, Pamukcu R, Thompson WJ: $\beta$-Catenin signaling. Cancer Biol Ther 2002, I:62I-625.

10. Lustig $B$, Behrens J: The Wnt signaling pathway and its role in tumor development. J Cancer Res Clin Oncol 2003, 129:199-221.

II. Endo Y, Rubin JS: Wnt signaling and neurite outgrowth: Insights and questions. Cancer Sci 2007, 98: I3।I-I3 I7.

12. Behrens J, von Kries JP, Kühl M, Bruhn L, Wedlich D, Grosschedl R, Birchmeier W: Functional interaction of beta-catenin with the transcription factor LEF-I. Nature 1996, 382:638-642.

13. Huber O, Korn R, McLaughlin J, Ohsugi M, Herrmann BG, Kemler R: Nuclear localization of beta-catenin by interaction with transcription factor LEF-I. Mech Dev 1996, 59:3-10.

14. He TC, Sparks AB, Rago C, Hermeking H, Zawel L, da Costa LT, Morin PJ, Vogelstein B, Kinzler KW: Identification of c-MYC as a target of the APC pathway. Science 1998, 28 I: 1509-I5/2.

15. Bettess MD, Dubois N, Murphy MJ, Dubey C, Roger C, Robine S, Trumpp A: c-Myc is required for the formation of intestinal crypts but dispensable for homeostasis of the adult intestinal epithelium. Mol Cell Biol 2005, 25:7868-7878.

16. Sherr C): Cancer cell cycles. Science 1996, 274:1672-1677.

17. Shtutman M, Zhurinsky J, Simcha I, Albanese C, D'Amico M, Pestell R, Ben-Ze'ev A: The cyclin DI gene is a target of the beta-catenin/LEF-I pathway. Proc Natl Acad Sci USA 1999, 96:5522-5527.

18. Filali $M$, Cheng N, Abbott D, Leontiev V, Engelhardt JF: Wnt-3A/ $\beta$ catenin signaling induces transcription from the LEF-I promotor. I Biol Chem 2002, 277:33398-334I0.

19. Uraguchi M, Morikawa M, Shirakawa M, Sanada K, Imai K: Activation of WNT family expression and signaling in squamous cell carcinomas of the oral cavity. J Dent Res 2004, 83:327-332.

20. Yap AS, Brieher WM, Gumbiner BM: Molecular and functional analysis of cadherin-based adherens junctions. Annu Rev Cell Dev Biol 1997, 13:1 19-146.

21. Bankfalvi A, Terpe HJ, Breukelmann D, Bier B, Rempe D, Pschadka G, Krech R, Lelle RJ, Boecker W: Immunophenotypic and prognostic analysis of $E$-cadherin and $\beta$-catenin expression during breast carcinogenesis and tumour progression: a comparative study with CD44. Histopathology 1999, 34:25-34.

22. Karayiannakis AJ, Nakopoulou L, Gakiopoulou H, Keramopoulos A, Davaris PS, Pignatelli M: Expression patterns of $\beta$-catenin in in situ and invasive breast cancer. Eur J Surg Oncol 200I, 27:3I-36.

23. Pirinen RT, Hirvikoski P, Johansson RT, Hollmen S, Kosma VM: Reduced expression of $\alpha$-catenin, $\beta$-catenin, and $\gamma$-catenin is associated with high cell proliferative activity and poor dif- ferentiation in non-small cell lung cancer. I Clin Pathol 200I, 54:39I-395

24. Lo Muzio L, Staibano S, Pannone G, Grieco M, Mignogna MD, Cerrato A, Testa NF, De Rosa G: Beta- and Gamma-catenin expression in oral squamaous cell carcinomas. Anticancer Res 1999, 19:3817-3826.

25. Tetsu O, McCormick $\mathrm{F}: \beta$-Catenin regulates expression of cyclin DI in colon carcinoma cells. Nature 1999, 398:422-426.

26. Kovesi G, Szende B: Prognostic value of cyclin DI, p27, and p63 in oral leukoplakia. J Oral Pathol Med 2006, 35:274-277.
Publish with Bio Med Central and every scientist can read your work free of charge

"BioMed Central will be the most significant development for disseminating the results of biomedical research in our lifetime. "

Sir Paul Nurse, Cancer Research UK

Your research papers will be:

- available free of charge to the entire biomedical community

- peer reviewed and published immediately upon acceptance

- cited in PubMed and archived on PubMed Central

- yours - you keep the copyright

Submit your manuscript here:

http://www.biomedcentral.com/info/publishing_adv.asp 